Full length article

\title{
Temperature independent refractive index measurement using a fiber Bragg grating on abrupt tapered tip
}

\author{
André D. Gomes ${ }^{\mathrm{a}, \mathrm{b}, *}$, Beatriz Silveira ${ }^{\mathrm{a}}$, Stephen C. Warren-Smith ${ }^{\mathrm{b}, \mathrm{c}}$, Martin Becker ${ }^{\mathrm{b}}$, Manfred Rothhardt ${ }^{\mathrm{b}}$, \\ Orlando Frazão ${ }^{a}$ \\ a INESC TEC and Department of Physics and Astronomy, Faculty of Sciences, University of Porto, Rua do Campo Alegre 687, 4169-007 Porto, Portugal \\ ${ }^{\mathrm{b}}$ Leibniz Institute of Photonic Technology IPHT, Albert Einstein Str. 9, 07745 Jena, Germany \\ ${ }^{\mathrm{c}}$ Institute for Photonics and Advanced Sensing (IPAS) and ARC Centre of Excellence for Nanoscale BioPhotonics (CNBP) and School of Physical Sciences, The University of \\ Adelaide, Adelaide, South Australia 5005, Australia
}

\section{A R T I C L E I N F O}

\section{Article history:}

Received 13 March 2017

Received in revised form 3 October 2017

Accepted 20 November 2017

\section{Keywords:}

Optical fiber sensors

Fiber Bragg grating

Abrupt taper tip

Refractive index sensing

Temperature independent measurements

\begin{abstract}
A B S T R A C T
A fiber Bragg grating was inscribed in an abrupt fiber taper using a femtosecond laser and phase-mask interferometer. The abrupt taper transition allows to excite a broad range of guided modes with different effective refractive indices that are reflected at different wavelengths according to Bragg's law. The multimode-Bragg reflection expands over $30 \mathrm{~nm}$ in the telecom-C-band. This corresponds to a modefield overlap of up to $30 \%$ outside of the fiber, making the device suitable for evanescent field sensing. Refractive index and temperature measurements are performed for different reflection peaks. Temperature independent refractive index measurements are achieved by considering the difference between the wavelength shifts of two measured reflection peaks. A minimum refractive index sensitivity of $16 \pm 1 \mathrm{~nm} /$ RIU was obtained in a low refractive index regime (1.3475-1.3720) with low influence of temperature $\left(-0.32 \pm 0.06 \mathrm{pm} /{ }^{\circ} \mathrm{C}\right)$. The cross sensitivity for this structure is $2.0 \times 10^{-5} \mathrm{RIU} /{ }^{\circ} \mathrm{C}$. The potential for simultaneous measurement of refractive index and temperature is also studied.
\end{abstract}

(c) 2017 Elsevier Ltd. All rights reserved.

\section{Introduction}

Fiber Bragg gratings (FBGs) are one of the most essential fiber sensing elements. They have been designed and developed for a wide variety of sensing applications, including: strain [1], temperature [2], pressure [3], and bending [4]. An FBG is a periodic modulation of the refractive index along the optical fiber, which is created by exposing the fiber core to an intense periodic optical beam, for example, using an ultraviolet femtosecond laser $[5,6]$. FBGs are generally created in photo-sensitive glass using an UV laser. In the case of optical fibers, hydrogen loading is needed for enhanced photosensitivity, since silica is non-photosensitive [7]. However, the use of femtosecond laser FBG inscription allows to address non-photosensitive optical materials such as optical fiber tapers. The focused ion beam technique is also used to create Bragg gratings in microfibers. Such structures can achieve sensitivities up to $660 \mathrm{~nm} / \mathrm{RIU}[8]$.

\footnotetext{
* Corresponding author at: INESC TEC and Department of Physics and Astronomy, Faculty of Sciences, University of Porto, Rua do Campo Alegre 687, 4169-007 Porto, Portugal.

E-mail address: adgomes@inesctec.pt (A.D. Gomes).
}

FBGs in conventional fiber are insensitive to changes in the external refractive index since they are not directly exposed to the outside medium. However, sensitivity can be gained by either thinning or etching the fiber before or after the FBG inscription [9], or by using suspended core microstructured optical fibers [10].

FBGs have been used for refractive index sensing for two decades, with numerous configurations proposed for different applications [11-19]. For example, FBG Fabry-Perot cavities incorporating microchannels show a linear refractive index response with sensitivities of $9 \mathrm{~nm} / \mathrm{RIU}$ between 1.43 and 1.49 for a broad microchannel, and $1 \mathrm{~nm} / \mathrm{RIU}$ between 1.3 and 1.7 for a narrow microchannel [13]. Fabry-Perot cavities based on high-birefringence FBGs can be used for refractive index and temperature sensing of liquids by monitoring the visibility as a function of refractive index and the wavelength shift as a function of temperature [14]. FBGs in microstructured optical fibers have also been studied for refractive index sensing [15-17]. A micro FBG for temperature compensated refractive index sensing was presented by Ran et al. [18]. Such sensor could achieve a maximum sensitivity of $-9.47 \mathrm{~nm} / \mathrm{RIU}$. Also for refractive index monitoring, an intensity sensor using a reflective tilted fiber Bragg grating was demonstrated using strong cladding to core recoupling [19]. This sensor is able to measure surrounding refractive index as low as 1.33 . 
In this paper, an FBG inscribed on an $18 \mu \mathrm{m}$ diameter abrupt tapered fiber tip is demonstrated for refractive index and temperature sensing. We show that it is possible to achieve temperature insensitive refractive index measurements by monitoring the difference between two reflection peaks. The proposed sensor is structurally and spectrally different, allowing to excite higher order modes than the sensor presented by Ran et al., obtaining greater refractive index sensitivity. Such a micro-sensor will have applications in bio-sensing, particularly where temperature fluctuations need to be considered.

\section{Fabrication and characterization}

The abrupt taper, with an $18 \mu \mathrm{m}$ diameter waist and a length of $40 \mathrm{~mm}$, was fabricated using a $\mathrm{CO}_{2}$ laser technique. In order to create the abrupt transition region, a constant tension was applied to the fiber from the beginning until the end of the whole taper production process. This tension is provided by the velocity difference of the translation stages in the $\mathrm{CO}_{2}$ laser system. To obtain an 18 $\mu \mathrm{m}$ diameter waist a leading stage velocity of $4000 \mu \mathrm{m} / \mathrm{s}$ and a rear stage velocity of $20 \mu \mathrm{m} / \mathrm{s}$ were used. Such constant tension created a taper transition region of approximately $300 \mu \mathrm{m}$ in length. Although it is long, a microfiber with an $18 \mu \mathrm{m}$ diameter waist is more resistant than a normal single mode fiber and presents high flexibility. Such mechanical properties are inherent to microfibers with diameters around this dimension or lower.

An FBG was inscribed in the abrupt taper using a phase-mask interferometer driven by a Ti:Sapphire laser with third harmonic generation providing femtosecond pulses in the UV range (267 $\mathrm{nm})$ [20]. Regarding the dimensions of the taper waist $(18 \mu \mathrm{m})$, the setup was adjusted to obtain a reflection wavelength of the fundamental mode near $1550 \mathrm{~nm}$ by using a diffraction mask with a period of $1075 \mathrm{~nm}$. Since the taper has an $18 \mu \mathrm{m}$ diameter waist it is mainly composed of pure silica ( $\mathrm{n}=1.444$ at $1550 \mathrm{~nm}$ ). Thus, it has low photosensitivity and a long inscription time of 10 min was used. A laser power of $200 \mathrm{~mW}$ was used during the inscription.
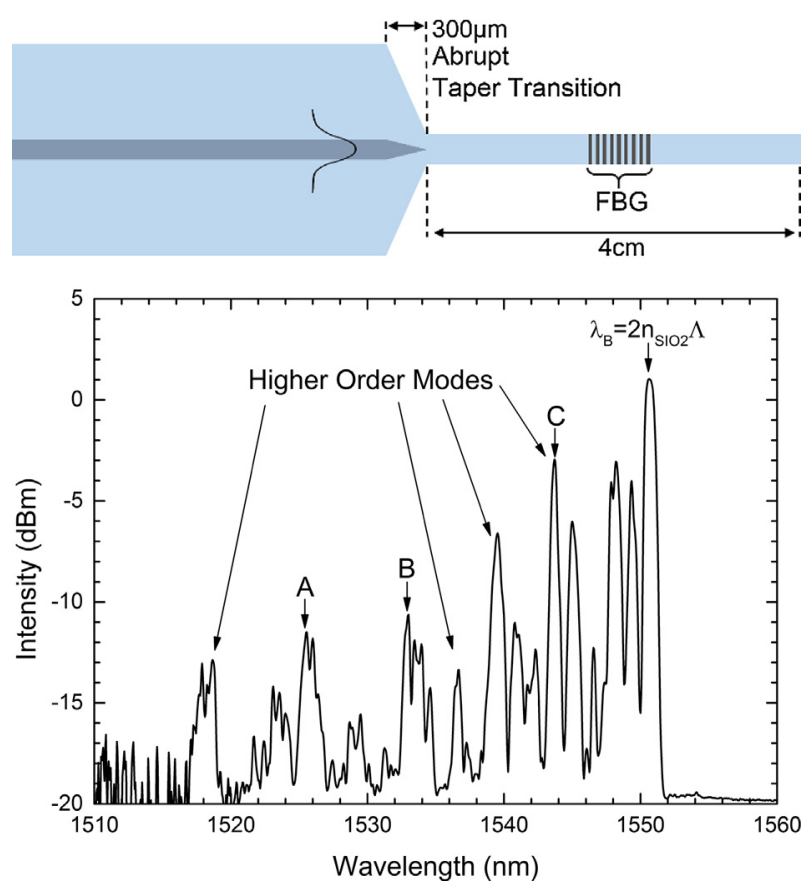

Fig. 1. Schematic of the sensor tip and its reflection spectrum in ethylene glycol (n $=1.3475$ ).
The taper was then broken at one of the transition regions in order to create the tip.

Fig. 1 shows a schematic of the FBG inscribed in the abrupt fiber taper tip and its reflection spectrum when immersed in an ethylene glycol solution $(n=1.3475)$. The sensor spectrum was measured using an optical circulator and a broadband source with a central wavelength of $1550 \mathrm{~nm}$ and a bandwidth of $100 \mathrm{~nm}$. The broadband source was connected to the optical circulator and the reflection spectrum was observed in an optical spectrum analyzer with $0.05 \mathrm{~nm}$ resolution. The fundamental mode peak is centered at $1550.6 \mathrm{~nm}$. The Bragg wavelength, $\lambda_{B}$, is given by:

$\lambda_{B}=2 n_{\text {eff }} \Lambda$

where $n_{\text {eff }}$ is the effective refractive index of the considered mode and $\Lambda$ is the Bragg grating period. Since the Bragg grating period is $537.5 \mathrm{~nm}$ and the reflection peak is centered at $1550.6 \mathrm{~nm}$, according to Eq. (1) the effective refractive index of the fundamental mode should be 1.4424 .

Due to the abrupt transition region of the taper tip, many modes with different effective refractive indices are excited providing reflections at different wavelengths. These reflection peaks would, in principle, extend until a Bragg wavelength of $1075 \mathrm{~nm}$, which correspond to the refractive index of air (a "mode" propagating in air). However, as seen in Fig. 1, the last mode excited is located at $1517.9 \mathrm{~nm}$ corresponding to an effective refractive index of 1.4120 , according to Bragg's law. The number of modes supported by the taper structure can be estimated using the generalized frequency $(\mathrm{V})$, which in this case is 38 . So, the number of modes supported by the taper region (estimated as $V^{2} / 2$ ) is around 722 . However, in reality, there is a certain higher order mode for which the power is at noise level. So, only a few modes carry enough power to be observed. The considered reflection peaks $\mathrm{A}$ and $\mathrm{B}$ are centered at $1525.52 \mathrm{~nm}$ and $1533 \mathrm{~nm}$, respectively with effective refractive indices of 1.4191 and 1.4260 .

For refractive index sensing, several solutions of ethylene glycol at different concentrations were used, resulting in a refractive index range from 1.3475 to 1.420 . The sensing tip was immersed in each solution and the wavelength shift for the fundamental mode and the higher order modes A, B, and C (see Fig. 1) were measured. Fig. 2(a) shows the reflection spectra under different refractive index solutions. The intensity of the higher order modes decreases as the solution refractive index increases. Regarding this, the higher order mode A was considered since peaks corresponding to lower effective indices decrease below the noise when measuring refractive index (i.e., $A$ is the last detectable peak at $n=$ $1.420)$. The wavelength shift as a function of refractive index for the four considered modes is depicted in Fig. 2(b). A polynomial fit was calculated for the higher order mode reflection peak $A$ $\left(121.7 \Delta n^{2}+18.5 n\right)$. The sensitivity is non-linear, but the higher order mode reflection peaks $B$ and $C$ can still be assumed as linear with a sensitivity of $11.4 \pm 0.4 \mathrm{~nm} / \mathrm{RIU}$ and $6.5 \pm 0.2 \mathrm{~nm} / \mathrm{RIU}$, respectively, in the range of refractive indices considered. For the fundamental mode, a sensitivity of $1.4 \pm 0.1 \mathrm{~nm} / \mathrm{RIU}$ was recorded.

Theoretically, the fundamental mode will be less sensitive to refractive index changes than higher order modes since the optical field is more confined within the taper, hence less affected by the external medium. A measurement of the amount of field that is inside of the taper can be obtained through the normalized propagation constant. The normalized propagation constant is given by [21]:

$b=\frac{n_{\text {eff }}^{2}-n_{\text {clad }}^{2}}{n_{\text {core }}^{2}-n_{\text {clad }}^{2}}$

where $n_{\text {eff }}$ is the effective refractive index, $n_{\text {core }}$ is the refractive index of the taper $\left(n_{\text {core }}=1.444\right)$ and $n_{\text {clad }}$ is the refractive index of 

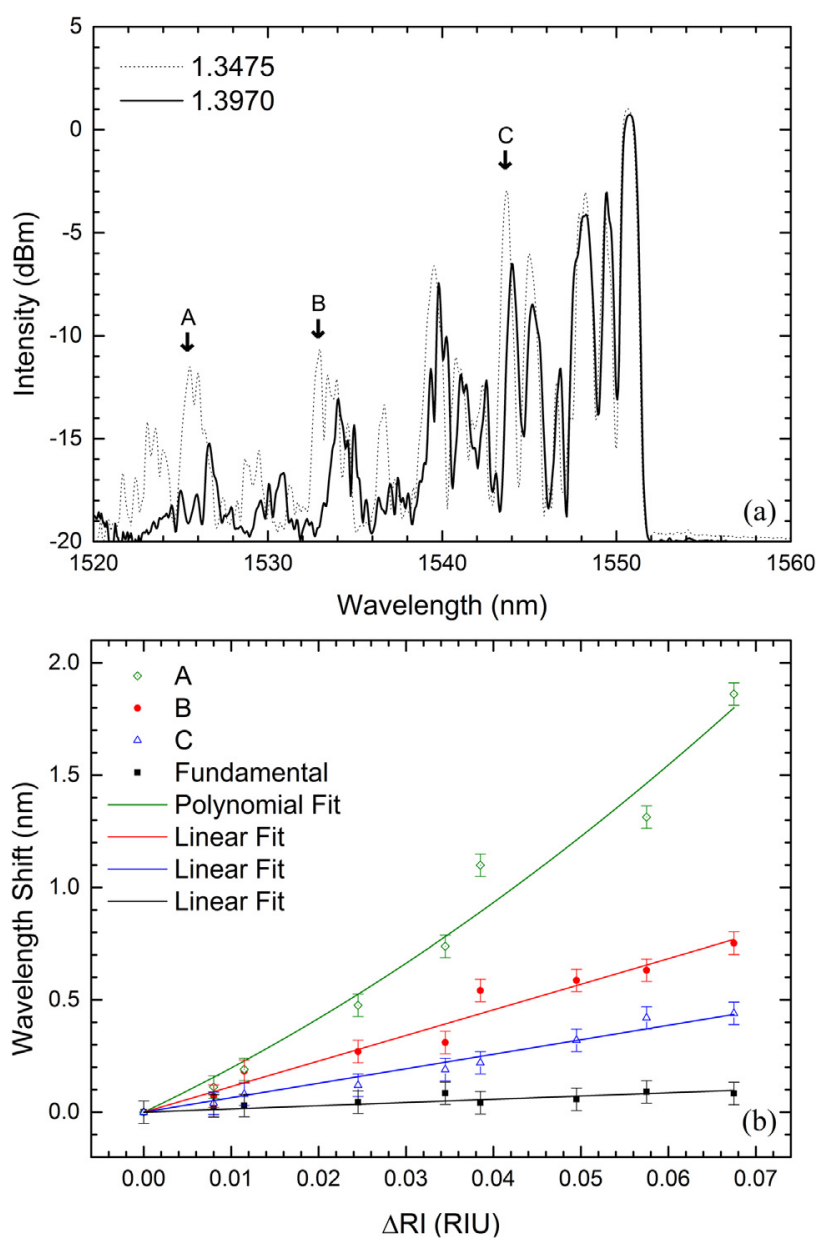

Fig. 2. (a) Reflection spectra of the sensor under two solutions with different refractive index solutions. (b) Wavelength shift as a function of refractive index variation for the fundamental mode and the higher order modes A, B and C. A quadratic fit was used for peak $A$, while the others can be assumed as linear within the considered refractive index range.

the external medium (in the case of Fig. $1, n_{\text {clad }}=1.3475$ ). Using the calculated effective refractive indices, the normalized propagation constant is 0.983 for the fundamental mode, 0.808 for the higher order mode B, and 0.735 for the higher order mode A. Under a refractive index of 1.3475 , only $2 \%$ of the electric field is outside of the taper in the fundamental mode, while in the higher order mode A almost $27 \%$ of the electric field is outside of the taper. The higher the order of the mode (the lower the effective index), the greater the contribution of the external medium to the effective refractive index of the mode (more electric field is outside of the fiber taper). Thus, each mode presents different sensitivity to the external parameters. Using such property the relative sensitivity between a higher order mode peak and a fundamental mode peak can be monitored. Moreover, the decrease in the higher order modes intensity as the external refractive index increases is related with the amount of electric field outside of the taper. As the external refractive index increases, the amount of electric field outside of the taper increases greatly for higher order modes, hence those modes present more losses. It is also noticeable that some resonant peaks deform while shifting, which in some cases may lead to difficulties in finding the exact position of the peak. Such behavior needs to be addressed in the future to improve the sensor stability.

The sensing tip was also studied in temperature from $30^{\circ} \mathrm{C}$ up to $120^{\circ} \mathrm{C}$. It is expected that the fundamental mode has higher sensitivity to temperature, since the mode is more confined and hence more affected by the thermo-optic coefficient of silica. In the same line of thought, higher order modes will have less sensitivity to temperature.

The higher order mode peak C (see Fig. 1) and the fundamental mode peak were monitored as a function of temperature. Fig. 3(a) shows the reflection spectra of the sensor in ethylene glycol solution ( $\mathrm{n}=1.3475$ ) at different temperatures. The wavelength shift as a function of temperature variation for both peaks is presented in Fig. 3(b). A linear fit was performed on the data, achieving a sensitivity of $9.6 \pm 0.1 \mathrm{pm} /{ }^{\circ} \mathrm{C}$ for the higher order mode peak $\mathrm{C}$ and a sensitivity of $10.0 \pm 0.1 \mathrm{pm} /{ }^{\circ} \mathrm{C}$ for the fundamental mode peak.

The higher order mode peaks $A$ and $B$ have temperature sensitivities of the same order of magnitude as the fundamental mode and peak $\mathrm{C}$. In order to reduce the temperature contribution, the wavelength shift difference between the fundamental mode and the considered higher order mode can be done. With this, one can use this sensor to measure refractive index with practically no influence of temperature variations and thermo-optic effects.

\section{Temperature independent refractive index sensing}

Considering $\Delta \lambda_{A}$ as the wavelength shift of the higher order mode reflection peak $A$ and $\Delta \lambda_{\text {Fund }}$ as the wavelength shift of the fundamental mode reflection peak, a new analysis can be made using the difference between both wavelength shifts, $\Delta \lambda_{A}-\Delta \lambda_{\text {Fund }}$. The difference between the wavelength shift of the reflection peak $A$ and the fundamental mode $\left(\Delta \lambda_{A}-\Delta \lambda_{\text {Fund }}\right)$ as a function of refractive index variation is shown in Fig. 4(a). Fig. 4
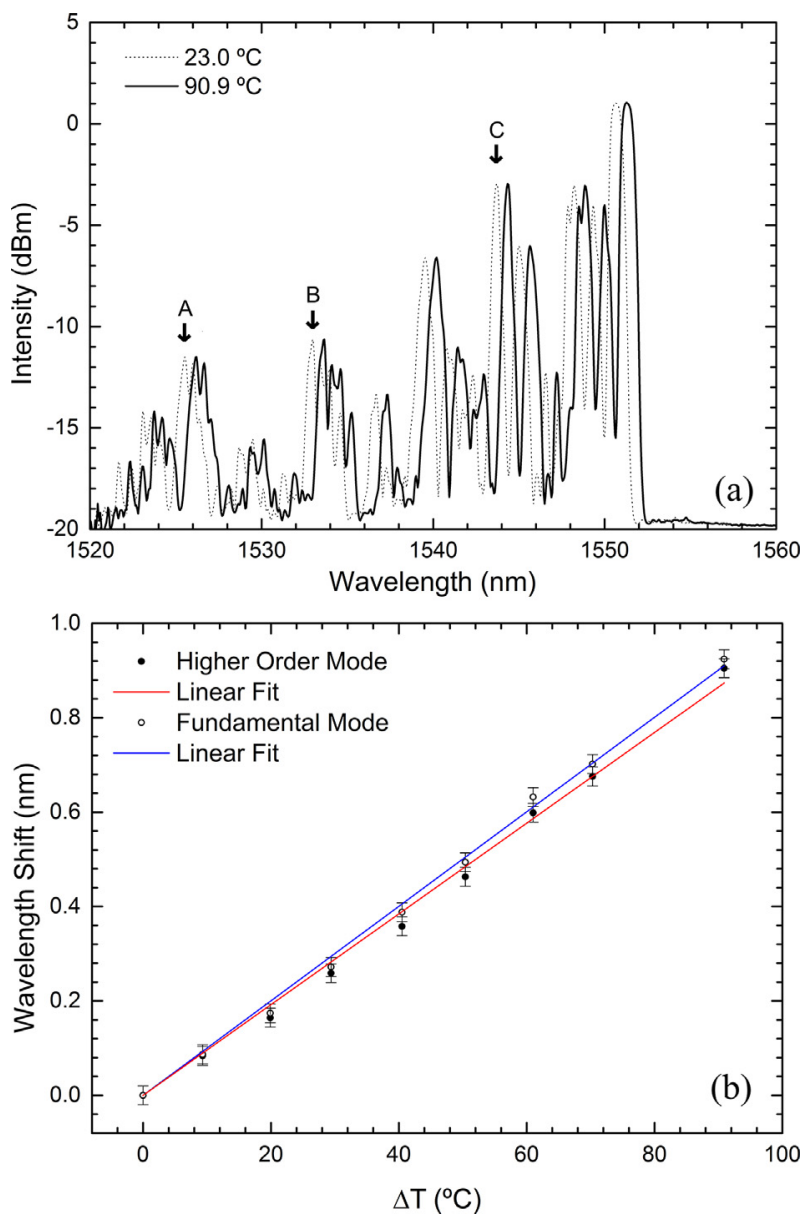

Fig. 3. (a) Reflection spectra of the sensor in ethylene glycol solution ( $\mathrm{n}=1.3475)$ at two different temperatures. (b) Wavelength shift as function of temperature variation for the fundamental mode peak and the higher order mode peak $\mathrm{C}$. 
(b) presents the difference between the higher order mode $\mathrm{C}$ wavelength shift and the fundamental mode wavelength shift as a function of temperature variation. Using a polynomial fit, the refractive index sensitivity with low temperature influence is given by $16.0 n+139.4 n^{2}$.

A linear refractive index sensitivity of $16 \pm 1 \mathrm{~nm} / \mathrm{RIU}$ can be considered in a low refractive index regime (1.3475-1.3720). In terms of temperature, a sensitivity of $-0.32 \pm 0.06 \mathrm{pm} /{ }^{\circ} \mathrm{C}$ is now obtained. Dividing both values, a cross-sensitivity of around 2.0 $\times 10^{-5} \mathrm{RIU} /{ }^{\circ} \mathrm{C}$ is obtained. A situation in which the temperature fluctuates $25^{\circ} \mathrm{C}$ during a refractive index measurement, the refractive index is only affected in the 4 th decimal place $( \pm 0.0005$ RIU).

\section{Simultaneous measurement of refractive index and temperature}

Feasibility analysis was performed on the simultaneous measurement of refractive index and temperature using this sensor. Such alternative way of measuring relies on the fact that both peaks have different sensitivities to each measured parameter. These sensitivities are used to create a matrix that is able to discriminate between the different measured parameters by analyzing the wavelength shift of each peak. Each reflection peak will suffer a wavelength shift due to refractive index and to temperature variations according to this relation:

$\Delta \lambda_{i}=\Delta n k_{\text {in }}+\Delta T k_{i T}$
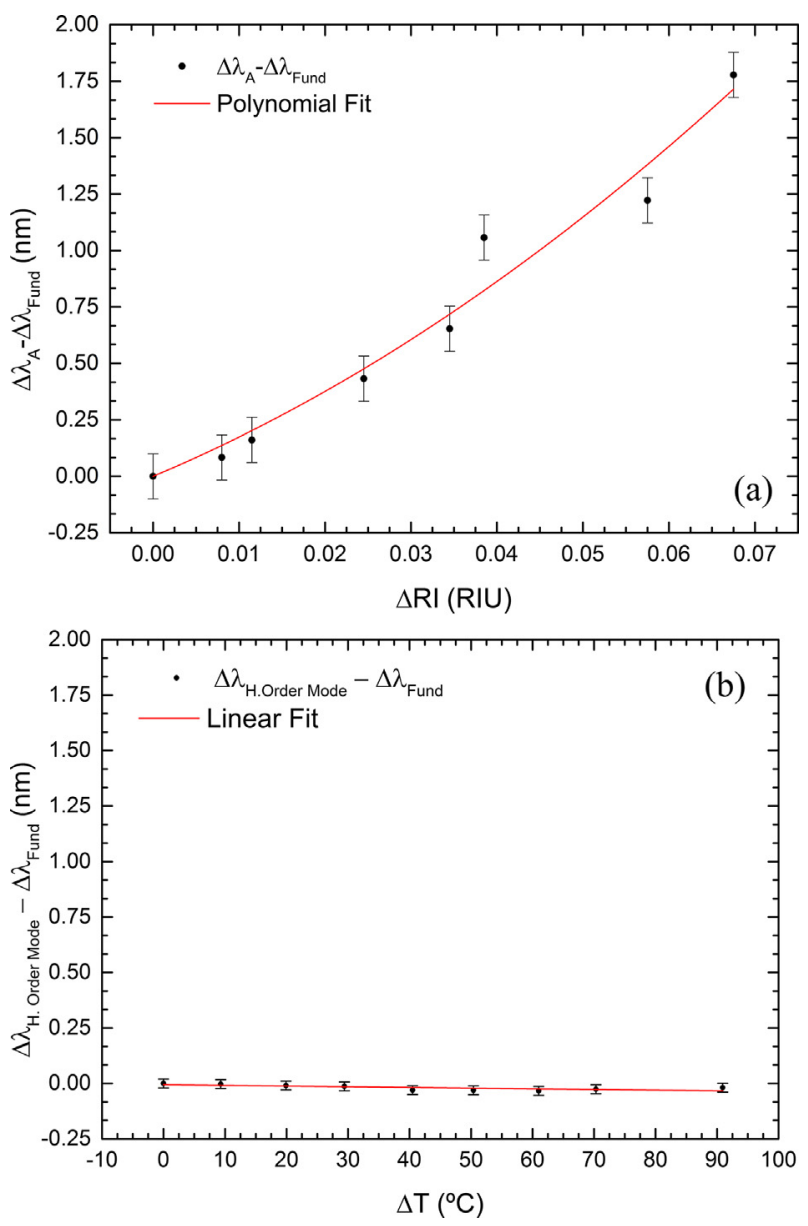

Fig. 4. (a) Difference between wavelength shifts $\left(\Delta \lambda_{A}-\Delta \lambda_{\text {Fund }}\right)$ as a function of refractive index variation. (b) Difference between wavelength shifts $\left(\Delta \lambda_{H}\right.$. Order Mode $\left.-\Delta \lambda_{\text {Fund }}\right)$ as a function of temperature variation. where $\Delta \lambda_{i}$ is the wavelength shift of the reflection peak " $i$ ", with $i=1,2, \Delta n$ and $\Delta T$ are respectively the refractive index and temperature variations, $k_{\text {in }}$ is the refractive index sensitivity of the reflection peak " $i$ " and $k_{i T}$ the temperature sensitivity of the reflection peak " $i$ ".

Considering two reflection peaks, the fundamental mode $\left(\lambda_{\text {Fund }}\right)$ and the higher order mode $C\left(\lambda_{C}\right)$, which is the most linear of the three considered higher order modes in terms of refractive index sensing, the expression involving the sensitivities of these peaks can be written in matrix form as:

$\left[\begin{array}{c}\Delta \lambda_{\text {Fund }} \\ \Delta \lambda_{C}\end{array}\right]=[K]=\left[\begin{array}{ll}k_{1 n} & k_{1 T} \\ k_{2 n} & k_{2 T}\end{array}\right]\left[\begin{array}{c}\Delta n \\ \Delta T\end{array}\right]$

Inverting the matrix expression to obtain the refractive index and temperature variations as a function of the wavelength shifts, one can obtain:

$$
\left[\begin{array}{c}
\Delta n \\
\Delta T
\end{array}\right]=\frac{1}{D}\left[\begin{array}{cc}
k_{2 T} & -k_{1 T} \\
-k_{2 n} & k_{1 n}
\end{array}\right]\left[\begin{array}{c}
\Delta \lambda_{\text {Fund }} \\
\Delta \lambda_{C}
\end{array}\right]
$$

where $D$ is the determinant of the matrix $[K], D=k_{1 n} k_{2 T}-k_{1 T} k_{2 n}$. From the values of sensitivity obtained before, Eq. (5) becomes:

$$
\left[\begin{array}{c}
\Delta n \\
\Delta T
\end{array}\right]=-\frac{1}{0.05156}\left[\begin{array}{cc}
0.0096 & -0.0100 \\
-6.5 & 1.4
\end{array}\right]\left[\begin{array}{c}
\Delta \lambda_{\text {Fund }} \\
\Delta \lambda_{C}
\end{array}\right]
$$

where $\Delta \lambda_{\text {Fund }}$ and $\Delta \lambda_{c}$ are given in nanometers, $\Delta n$ in refractive index units, and $\Delta T$ in degrees Celsius. The stability of this method can be verified through the condition number of the matrix, which is a quantity that provides a qualitative estimate for the sensitivity of the solution of a system of linear equations [16]. The condition number is given by:

$$
\text { Condition } \#=\|K\|_{2}\left\|K^{-1}\right\|_{2}
$$

In this case, where we have a square matrix, the Euclidian norm can be used:

$$
\|K\|_{2}=\sqrt{\lambda_{\max }\left(K^{*} \cdot K\right)}
$$

where $\lambda_{\max }$ is the largest eigenvalue of the matrix $K^{*} \cdot K\left(\mathrm{~K}^{*}\right.$ is the conjugate transpose of $K$ ). The lower the condition number, the lower the error. For high condition number matrices, a small change in the wavelength shifts will lead to a large error in the solution. In this case, the condition number is 997, which shows that the matrix is ill-conditioned. That is, the sensor in the particular configuration presented here is not well suited to simultaneous measurement of temperature and refractive index. This value could be improved if the temperature and refractive index sensitivities were of the same order of magnitude.

\section{Conclusion}

An FBG was inscribed in an abrupt taper using a phase-mask interferometer driven by a femtosecond laser. The inscribed FBG had a period of $537.5 \mathrm{~nm}$, which corresponds to a Bragg wavelength of $1550.6 \mathrm{~nm}$ for the fundamental mode of the abrupt taper. Due to the abrupt taper transition, many modes are excited with different effective refractive indices that reflected at different wavelengths according to Bragg's law. The lowest wavelength measured in the structure corresponds to a Bragg wavelength of $1517.9 \mathrm{~nm}$.

Refractive index and temperature measurements were performed using this sensor. For refractive index sensing the wavelength shift of three higher order modes and the fundamental mode were studied. For the higher order mode A, a quadratic behavior is observed $\left(121.7 \Delta n^{2}+18.5 \Delta n\right)$, while the higher order modes $\mathrm{B}$ and $\mathrm{C}$ can still be assumed approximately as linear. A 
refractive index sensitivities of $11.4 \pm 0.4 \mathrm{~nm} / \mathrm{RIU}$ and $6.5 \pm 0.2 \mathrm{~nm} /$ RIU were achieved respectively for the higher order mode B and C in the refractive index range from 1.3475 to 1.420 . The fundamental mode is less sensitive to refractive index, with a sensitivity of $1.4 \pm 0.1 \mathrm{~nm} / \mathrm{RIU}$. It is clearly visible that the higher the order of the mode, the greater the sensitivity. Modes of higher order than the ones considered for the measurements here should have more sensitivity to refractive index since the optical field is more affected by the external medium (the mode is less confined).

For temperature sensing, a sensitivity of $9.6 \pm 0.1 \mathrm{pm} /{ }^{\circ} \mathrm{C}$ was achieved for the higher order mode peak $C$. As expected, the highest sensitivity was obtained for the fundamental mode (10.0 \pm 0.1 $\mathrm{pm} /{ }^{\circ} \mathrm{C}$ ) since the mode is more confined and more affected by the thermo-optic coefficient of silica.

Considering the difference between the wavelength shifts of both measured peaks, it is possible to measure refractive index with low influence of temperature variations (within the order of $-0.32 \mathrm{pm} /{ }^{\circ} \mathrm{C}$ ) and thermo-optic effects since the measurement already includes the contribution of the liquid refractive index variations due to temperature. Considering a low refractive index range (from 1.3475 to 1.3720 ), where the sensitivity is relatively low, the refractive index sensitivity is $16 \pm 1 \mathrm{~nm} / \mathrm{RIU}$. This shows a minimum refractive index sensitivity $60 \%$ higher than the maximum sensitivity reported previously by Ran et al. [18]. In this paper, a $\mathrm{CO}_{2}$ laser system was used, which allowed the fabrication of highly abrupt tapers. We have reduced the transition length of the taper from $2 \mathrm{~mm}$ reported in Ran et al. [18] to $300 \mu \mathrm{m}$. This allows relatively higher order modes to be excited and thus greater sensitivities to be achieved. The cross sensitivity for this method is $2.0 \times 10^{-5} \mathrm{RIU} /{ }^{\circ} \mathrm{C}$, which for variations of $25^{\circ} \mathrm{C}$ the refractive index sensing is only affected in the 4 th decimal place. To achieve higher refractive index sensitivities, one should be able to excite higher order modes with lower normalized propagation constants (lower effective index) than the one considered in this paper.

A feasibility analysis was performed for the simultaneous measurement of refractive index and temperature using a matrix method to discriminate between these two parameters. It was determined that the matrix is ill-conditioned for the parameters used, which is due to the fact that the temperature sensitivities of both modes are similar and the values are orders of magnitude lower than the refractive index sensitivity values. The sensor has potential to be used in medical applications, where temperature changes are low. Moreover, the proposed sensor is monitored in reflection, while most of the tilted fiber Bragg grating configurations need to be monitored in transmission. However, these sensors can now operate in reflection mode by adding a broadband mirror at the end of the tilted fiber Bragg grating. This can be achieved through silica/air Fresnel reflection by simply cleaving the fiber end, or by depositing a metal coating on the fiber end [22]. In future, a greater discrimination in temperature sensitives could be achieved by exciting even higher order modes or reducing the microfiber diameter, which may allow this method to be successfully implemented. Mode analysis will also be carried out in the future for better understanding of the reflection spectra.

\section{Acknowledgement}

André Gomes was funded by DAAD (German Academic Exchange Service) through the short-term grant 57214227/2016 from March to July 2016. He is currently funded by FCT (PD/ BI/129000/2017). This work was financed by FCT - Fundação para a Ciência e Tecnologia (Portuguese Foundation for Science and Technology) and by ERDF (European Regional Development Fund) through: COMPETE Programme (Operational Programme for Competitiveness) within project FCOMP-01-0124-FEDER-037281; ON.2 - O Novo Norte (Northern Portugal Regional Operational Programme). Stephen Warren-Smith was funded by the European Commission through the Seventh Framework Programme (FP7), PIIF-GA-2013-623248 from February 2015 to September 2016. He is currently funded by a Ramsay Fellowship from the University of Adelaide.

\section{References}

[1] O. Frazão, S.F.O. Silva, A. Guerreiro, J.L. Santos, L.A. Ferreira, F.M. Araújo, Strain sensitivity control of fiber Bragg grating structures with fused tapers, Appl. Opt. 46 (36) (2007) 8578-8582.

[2] D.a. Pereira, O. Frazão, J.L. Santos, Fiber Bragg grating sensing system for simultaneous measurement of salinity and temperature, Opt. Eng. 43 (2004) 299-304.

[3] C.J.S. De Matos, P. Torres, L.C.G. Valente, W. Margulis, R. Stubbe, Fiber Bragg grating (FBG) characterization and shaping by local pressure, J. Light. Technol. 19 (8) (2001) 1206-1211.

[4] Y.X. Jin, C.C. Chan, X.Y. Dong, Y.F. Zhang, Temperature-independent bending sensor with tilted fiber Bragg grating interacting with multimode fiber, Opt. Commun. 282 (19) (2009) 3905-3907.

[5] J. Chen, B. Liu, H. Zhang, Review of fiber Bragg grating sensor technology, Front. Optoelectron. Chin. 4 (2) (2011) 204-212.

[6] K.O. Hill, G. Meltz, Fiber Bragg grating technology fundamentals and overview, J. Light. Technol. 15 (8) (1997) 1263-1276.

[7] P.J. Lemaire, R.M. Atkins, V. Mizrahi, W.a. Reed, High pressure $\mathrm{H} \_2$ loading as a technique for achieving ultrahigh UV photosensitivity and thermal sensitivity in $\mathrm{GeO} 2$ doped optical fibres, Electron. Lett. 29(13) (1993) 1191-1193.

[8] Y. Liu, C. Meng, a.P. Zhang, Y. Xiao, H. Yu, L. Tong, Compact microfiber Bragg gratings with high-index contrast, Opt. Lett. 36(16) (2011) 3115-3117.

[9] W. Liang, Y. Huang, Y. Xu, R.K. Lee, A. Yariv, Highly sensitive fiber Bragg grating refractive index sensors, Appl. Phys. Lett. 86 (15) (2005) 1-3.

[10] M. Becker et al., Inscription of fiber Bragg grating arrays in pure silica suspended core fibers, IEEE Photon. Technol. Lett. 21 (19) (2009) 1453-1455.

[11] G. Meltz, S.J. Hewlett, J.D. Love, D.H. Road, Fiber grating evanescent-wave sensors, 2836 (1996) 342-350.

[12] R.A. Asseh, S. Sandgren, H. Ahlfeldt, B. Sahlgren, G.E. Stubbe, A. Asseh, S. Sandgren, H.B. Ahlfeldt, Fiber optical Bragg grating refractometer, Fiber Integr. Opt. 17(1) (1998) 51-62.

[13] K. Zhou, Z. Yan, L. Zhang, I. Bennion, Refractometer based on fiber Bragg grating Fabry-Pérot cavity embedded with a narrow microchannel, Opt. Exp. 19 (12) (2011) 11769-11779.

[14] C. Gouveia, P.A.S. Jorge, J.M. Baptista, O. Frazão, Fabry-Pérot cavity based on a high-birefringent fiber Bragg grating for refractive index and temperature measurement, IEEE Sens. J. 12 (1) (2012) 17-21.

[15] M.C. Phan Huy et al., Three-hole microstructured optical fiber for efficient fiber Bragg grating refractometer, Opt. Lett. 32 (16) (2007) 2390-2392.

[16] M.C. Phan Huy et al., Tilted fiber Bragg grating photowritten in microstructured optical fiber for improved refractive index measurement, Opt. Exp. 14 (22) (2006) 10359-10370.

[17] S.C. Warren-Smith, T.M. Monro, Exposed core microstructured optical fiber Bragg gratings: refractive index sensing, Opt. Exp. 22 (2) (2014) 1480-1489.

[18] Y. Ran, L. Jin, L. Sun, J. Li, B. Guan, Temperature-compensated refractive-index sensing using a single Bragg grating in an abrupt fiber taper, IEEE Photon. J. 5 (2) (2013) 7100208 .

[19] T. Guo, H. Tam, P.A. Krug, J. Albert, Reflective tilted fiber Bragg grating refractometer based on strong cladding to core recoupling, Opt. Exp. 17 (7) (2009) 5736-5742.

[20] M. Becker et al., Fiber Bragg grating inscription combining DUV subpicosecond laser pulses and two-beam interferometry, Opt. Exp. 16 (23) (2008) 19169-19178.

[21] C. Chen, Foundations for Guided-wave Optics, John Wiley \& Sons, 2006.

[22] C.-F. Chan, C. Chen, A. Jafari, A. Laronche, D.J. Thomson, J. Albert, Optical fiber refractometer using narrowband cladding-mode resonance shifts, Appl. Opt. 46 (7) (2007) 1142. 